\title{
Aggressive B-Cell Lymphoma with Dual Surface Immunoglobulin Light-Chain Expression
}

\author{
Tohru Fujiwara ${ }^{1}$, Kenichi Ishizawa ${ }^{2}$, Katsura Kohata ${ }^{1}$, Joji Yamamoto ${ }^{1}$, Minami F Yamada ${ }^{1}$, \\ Junichi Kameoka ${ }^{1}$, Ryo Ichinohasama ${ }^{2}$ and Hideo Harigae ${ }^{1}$
}

\begin{abstract}
Dual surface immunoglobulin light-chain expression in B-cell malignant neoplasm is a rare event, and has been predominantly reported in chronic lymphocytic leukemia. Herein, we report a case of aggressive B-cell lymphoma with $\kappa / \lambda$-dual surface immunoglobulin light-chain expression of a 69 -year-old woman. The lymphoma cells were positive for CD5, CD19, CD20, HLA-DR, Igא and Ig $\lambda$. Southern blot analysis confirmed rearranged bands for both light chains with a monoclonal heavy chain rearrangement. She was treated with a combination of rituximab and CHOP regimen, but died of the progressive disease. To our knowledge, this is the first case of aggressive B-cell lymphoma showing dual $\kappa / \lambda$ expression; the possible mechanisms of abnormal light chain expression are discussed.
\end{abstract}

Key words: aggressive B-cell lymphoma, $\kappa / \lambda$ dual expression, gene rearrangement

(DOI: 10.2169/internalmedicine.46.0128)

\section{Introduction}

Mature B lymphocytes typically express either $\kappa$ or $\lambda$ light-chain, a restriction known as allelic exclusion. Whereas $99.99 \%$ of B cells have allelic exclusion at the IgH locus in mice (1), there are few data to draw from to estimate the extent of allelic exclusion at the IgL locus. Indeed, there have been several examples that report on light-chain double producers in normal peripheral B cells as well as in B-cell malignant neoplasms including chronic lymphocytic leukemia (CLL) (2). However, probably because of the limited number of reports available to date, the cellular and molecular mechanisms of dual light-chain expression have not been completely elucidated. Herein, we report a case of B-cell lymphoma with dual surface immunoglobulin light-chain expression, which pursued a clinically aggressive course.

\section{Case Report}

A 69-year-old Japanese woman was admitted to our hospital in July 2006 because of high fever and chills persisting for 4 weeks. She had a past history of cerebral infarction.
She had massive splenomegaly with no obvious lymphadenopathy. Hematological data was as follows: hemoglobin 7.2 $\mathrm{g} / \mathrm{dL}$, platelets $95 \times 10^{9} / \mathrm{L}$ and white blood cells (WBC) $12.1 \times$ $10^{9} / \mathrm{L}$ with $94 \%$ neutrophils, $5 \%$ lymphocytes, $1 \%$ myeloblast. Serum levels of lactate dehydrogenase (LDH), C-reactive protein, soluble interleukin-2 receptor (sIL-2R) and ferritin were $1680 \mathrm{IU} / \mathrm{L}, 2.1 \mathrm{mg} / \mathrm{dL}, 9882 \mathrm{U} / \mathrm{mL}$ and $2977 \mathrm{ng} / \mathrm{mL}$, respectively. Serum antibody titers did not indicate the presence of autoimmune disease as well as any primary or continuing infection with cytomegalovirus and Epstein-Barr virus. Bone marrow examination disclosed infiltration of abnormal lymphoid cells (15.8\% of all nucleated cells) with a high degree of nuclear atypia and distinct nucleoli. Mature histiocytes phagocytosing various hematopoietic cells were also observed. Surface marker analysis of bone marrow mononuclear cells revealed that they were positive for CD5 (55\%), CD19 (68\%), CD20 (74\%) and HLA-DR (72\%). Notably, the lymphoma cells were positive for both $\operatorname{IgK}(97 \%)$ and $\operatorname{Ig} \lambda(89 \%)$ based on a CD19+ gate strategy (Fig. 1A). Rearrangement bands for immunoglobulin heavy chain $(\mathrm{JH})$ and both light-chain genes $(\mathrm{J} \kappa, \mathrm{C} \lambda)$ were detected by Southern blot analyses, while only a germline band with decreased intensity was observed when $\mathrm{C \kappa}$

${ }^{1}$ Department of Rheumatology and Hematology, Tohoku University Graduate School of Medicine, Sendai and ${ }^{2}$ Hematopathology, Tohoku University Graduate School of Medicine, Sendai

Received for publication February 27, 2007; Accepted for publication May 21, 2007

Correspondence to Dr. Tohru Fujiwara, fujiwara-t@mail.tains.tohoku.ac.jp 
(a)

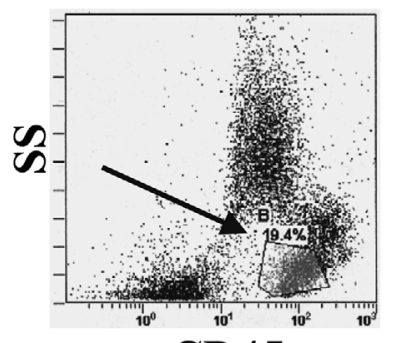

CD45
B

(a) JH

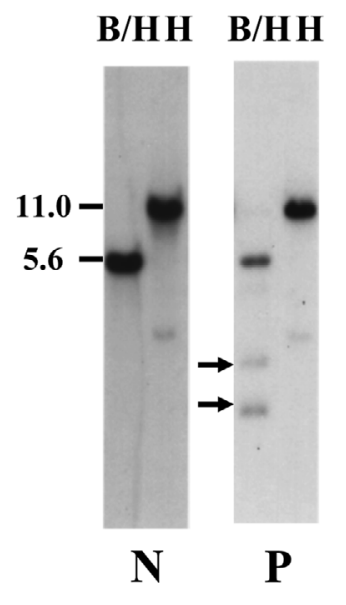

(b)

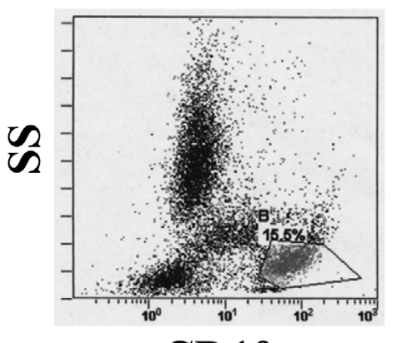

CD19 (c)

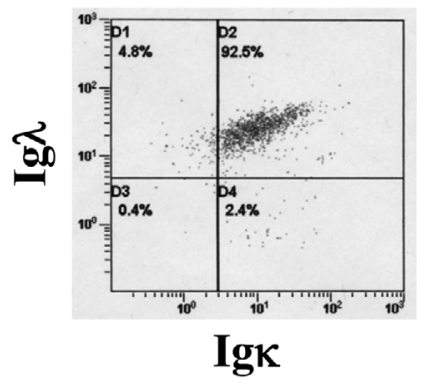

(b) $\mathbf{J} \mathbf{K}$

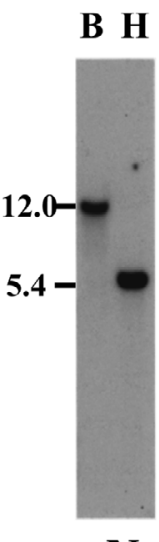

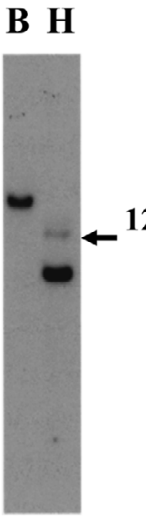

P (c) $\mathrm{CK}$

(d) $\mathrm{C} \lambda$

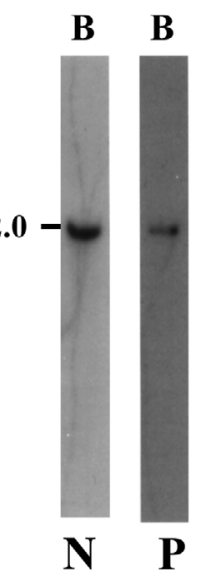

E/H E/H

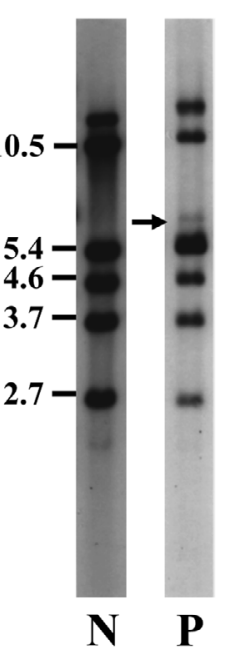

Figure 1. Flow cytometric and genetic analyses of the dual $\kappa / \lambda$-positive lymphoma cells. (A) (a) Bone marrow cells were sorted by a $\mathrm{CD}^{+}$gate procedure using Cytomics FC 500 (BECKMAN COULTER, Tokyo, Japan) and an abnormal cell population, which showed positivity for CD5 and CD20, was detected (arrow). (b, c) Among them, the $\mathrm{CD} 19^{+}$fraction was examined for the expression of IgK and $\operatorname{Ig} \lambda$. Antibodies recognizing human kappa $F\left(a^{\prime}\right)_{2}$ and lambda $F\left(a^{\prime}\right)_{2}$ were purchased from BD Biosciences (San Jose, CA, USA). (B) Southern blot analyses of bone marrow cells using probes for immunoglobulin heavy chain (a; JH) and both light-chain genes (b; J $\kappa$, c; $\mathrm{C} \kappa$ and d; $\mathrm{C} \lambda$ ) are shown. Arrow indicates the rearranged bands. N; normal sample, P; patient sample, B/H; BamHI/HindIII, H; HindIII, B; BamHI, E/H; EcoRI/HindIII.

probe was used for the analysis (Fig. 1B). Cytogenetic analysis of bone marrow cells showed the complex abnormality, with representative karyotype of $46, \mathrm{XX},-1,-1,-2$, 3, add (4) (p14), del (7) (q34), -8, add (9) (p13), add (11) (p 11), -14, -14, del (15) (q22), +mar1, +mar2, +mar3, +mar4, +mar5, +mar6, +mar7 (Fig. 2). Thus, she was diagnosed as having B-cell non-Hodgkin's lymphoma associated with hemophagocytic syndrome (HPS). The sites of involvement, clinical features and pathological findings suggested that the lymphoma cell might be categorized into either diffuse large B-cell lymphoma or intravascular large B-cell lymphoma according to the World Health Organization Classification. She was treated with R-CHOP regimen, which initially showed a good clinical response. However, she had the complication of acute cholangitis in September 2006, which was the obstacle in conducting further chemotherapy. In December 2006, the patient relapsed and died of progressive disease.

\section{Discussion}

We report a unique case of B-cell lymphoma showing $\mathrm{\kappa} /$ $\lambda$-dual expression on the cell surface, with rearrangements in both light chain genes on Southern blot analysis. Although the underlying molecular mechanisms for the generation of $\kappa / \lambda$-positive clones have not been completely elucidated, three assumptions have been suggested to date (2); 1) 


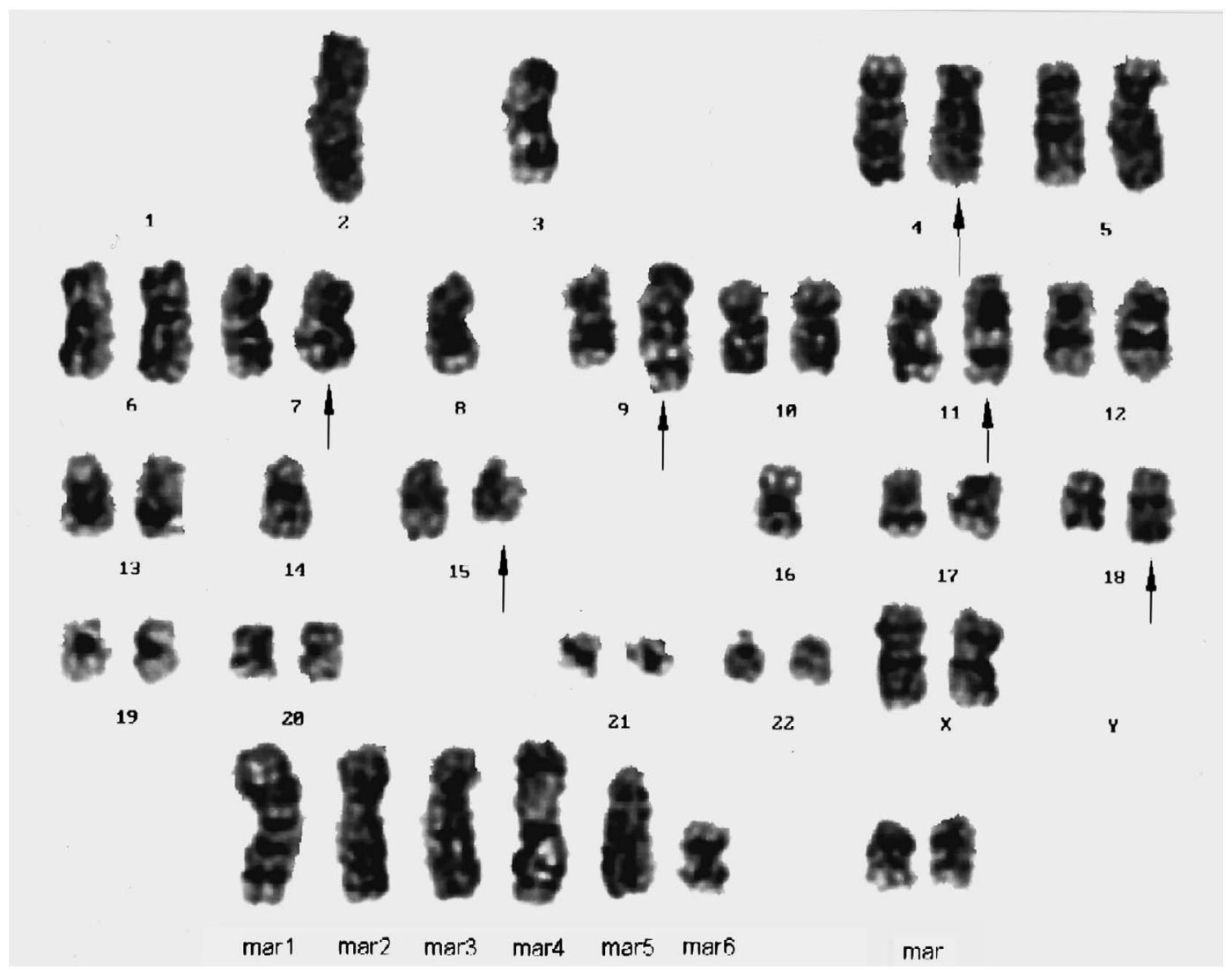

Figure 2. G-banded karyotype of lymphoma cells: 46,XX, -1, -1, -2, -3, add(4)(p14), del(7)(q34), 8, $\operatorname{add}(9)(p 13), \operatorname{add}(11)(p 11),-14,-14, \operatorname{del}(15)(q 22),+$ mar1, +mar2, +mar3, +mar4, +mar5, +mar6, +mar7 [1], 47, idem, +mar [1], 46, idem, +8, -add(11), -mar7, +mar [2], 47, idem, -add(9), -13, +14, $\operatorname{add}(18)(q 23),-m a r 7,+\operatorname{mar} 3$ [1], 47, idem, $-\operatorname{add}(9),+\operatorname{add}(9)(q 34),+14,-16, \operatorname{add}(18)(q 23),-\operatorname{mar} 7$, +2mar [1], 47, idem, -X, -add(9), +add(9)(q34), +14, add(18)(q23), -mar6, -mar7, +3mar [1], 46, XX [3]. Metaphases were G-banded by a trypsin-treated Giemsa stain. Abberant chromosomes are indicated by arrows.

a failure to destroy expression of the original $\mathrm{L}$ gene during a secondary light-chain rearrangement [receptor editing (3)] , 2) a clonal progression from a single rearranged light-chain into dual light-chain expressing cells, 3) simultaneous rearrangements at both light-chain loci. Among them, we speculated that a failure of receptor editing might underlie the generation of dual $\kappa / \lambda$ positive clones in the present case, although the data available were too limited. In general, $\kappa$ rearrangements are favored over $\lambda$ rearrangements, and $\kappa$ loci in $\lambda$ producers are often rearranged and inactivated by rearrangements of the kappa-deletion element (KDE) $(3,4)$. However, it has also been reported that such processes occasionally fail to destroy the original $\kappa$ lightchain gene $(2,3)$. Thus, it might be possible to consider that a moderately decreased germline band detected by $\mathrm{C} \kappa$ probe (Fig. 1B-c) might imply the presence of rearrangement involving KDE to heptamer-nonamer recombination signal sequences, which causes the deletion of $\mathrm{C} \kappa$ locus as well as Igא enhancer (5). However, in the present case, this rear- rangement might not be sufficient to inactivate the transcription of Ig $\kappa$ gene, thereby creating dual $\kappa / \lambda$ light-chain positive clones. This point warrants further investigation.

Of interest is that the present case displayed a clinically aggressive course, whereas the previous case reports of $\kappa / \lambda$ dual expression in B-cell neoplasm were predominantly observed in indolent cases comprising CLL (2, 6-8). Unfortunately, there has been no evidence available demonstrating the biological properties of dual $\kappa / \lambda$ producers, which would support our observations. In addition, we could not exclude the possibility that the clinical aggressiveness in the present case might be related to the underlying pathological features (aggressive B-cell lymphoma), and the coexistence of B-cell lymphoma-associated HPS, rather than dual $\kappa / \lambda$ rearrangement/expression. Therefore, further investigations into similar cases would be necessary to clarify the molecular mechanism and the clinical significance of double light-chain producers.

\section{References}


DOI: $10.2169 /$ internalmedicine.46.0128

2001.

2. Xu D. Dual surface immunoglobulin light-chain expression in Bcell lymphoproliferative disorders. Arch Pathol Lab Med 130: 853-856, 2006.

3. Gerdes T, Wabl M. Autoreactivity and allelic inclusion in a B cell nuclear transfer mouse. Nat Immunol 5: 1282-1287, 2004.

4. Brauninger A, Goossens T, Rajewsky K, Kuppers R. Regulation of immunoglobulin light chain gene rearrangements during early B cell development in the human. Eur J Immunol 31: 3631-3637, 2001.

5. Beishuizen A, Verhoeven MA, Mol EJ, van Dongen JJ. Detection of immunoglobulin kappa light-chain gene rearrangement patterns by Southern blot analysis. Leukemia 8: 2228-2236, 1994.

6. del Senno L, Gandini D, Gambari R, Lanza F, Tomasi P, Castoldi G. Monoclonal origin of $\mathrm{B}$ cells producing $\kappa$, lambda and $\kappa$ lambda immunoglobulin light chains in a patient with chronic lymphocytic leukemia. Leuk Res 11: 1093-1098, 1987.

7. Peltomaki P, Bianchi NO, Knuutila S, et al. Immunoglobulin kappa and lambda light chain dual genotype rearrangement in a patient with kappa-secreting B-CLL. Eur J Cancer Clin Oncol 24: 1233-1238, 1988.

8. Kawada H, Fukuda R, Yoshida M, et al. A novel variant of Blymphoid leukemia expressing kappa/lambda light chains. Acta Haematol 100: 54-56, 1998.

(C) 2007 The Japanese Society of Internal Medicine http://www.naika.or.jp/imindex.html 